\begin{tabular}{l|l|l}
\hline 1903 & $\Delta a$ & $\Delta \delta$ \\
\hline
\end{tabular}

(347) Pariana.

[A. N. 3868 . G. Boccardi].

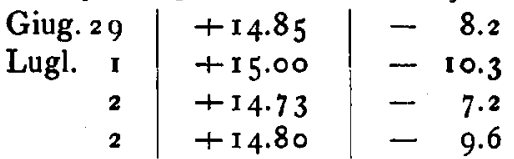

La rifrazione già computata in questi $\mathrm{O}-\mathrm{C}$ ha $\mathrm{i}$ seguenti valori:

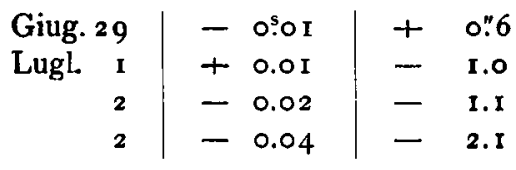

(7I) Niobe.

[B. A. J. 1905].

Nov. I 2 - $15.08 \mid-104.6$

Arcetri-Firenze, 1904 Giugno.

\begin{tabular}{|c|c|c|}
\hline 1903 & $\Delta \alpha$ & $\Delta \delta$ \\
\hline \multicolumn{3}{|c|}{$\begin{array}{l}\text { (28) Bellona. } \\
\text { [B. A. J. I905]. }\end{array}$} \\
\hline \multicolumn{3}{|c|}{$-404^{5} 80 \mid-1$} \\
\hline \multicolumn{3}{|c|}{$\begin{array}{l}(324) \text { B a mberga. } \\
{[\text { Eff. man. Berberich].*) }}\end{array}$} \\
\hline Agos. 7 & -0.42 & -0.7 \\
\hline 18 & -0.39 & -0.5 \\
\hline 20 & $-0.7^{2}$ & +2.9 \\
\hline $2 \mathrm{I}$ & $-0.8 \mathrm{I}$ & $+\mathbf{r} \cdot 1$ \\
\hline 22 & -0.70 & +0.8 \\
\hline 24 & -0.84 & 0.0 \\
\hline 24 & -0.40 & +4.5 \\
\hline 25 & -0.82 & -0.4 \\
\hline 27 & -0.45 & +2.1 \\
\hline 27 & -0.65 & $-\mathrm{T} .3$ \\
\hline
\end{tabular}

\begin{tabular}{r|c|c}
\hline \multicolumn{1}{c|}{1903} & $\Delta \alpha$ & $\Delta \delta$ \\
\hline Agos.28 & -0.64 & $+1 ! 3$ \\
28 & -0.73 & -0.8 \\
29 & -0.58 & -0.2 \\
30 & -0.65 & -2.9 \\
31 & -0.55 & -0.9 \\
Sett. 1 & -0.50 & -1.5 \\
2 & -0.59 & -2.0 \\
18 & -0.37 & -3.8 \\
18 & -0.50 & -2.1 \\
18 & -0.50 & -5.1 \\
18 & -0.50 & -4.0 \\
19 & -0.55 & -4.7 \\
19 & -0.54 & -3.4 \\
20 & -0.65 & -4.0 \\
20 & -0.45 & -2.1 \\
23 & -0.47 & -3.2 \\
24 & -0.22 & -2.0 \\
24 & -0.40 & -3.5
\end{tabular}

A. Abetti.

*) L' effemeride approssimata si trova in A. N. 3868. Gli O-C quì pubblicati mi furono gentilmente favoriti dal prof. Berberich. Sottoposti a rigoroso esame, specialmente rispetto alla bontà delle posizioni stellari, si venne alla conclusione che se non si possono fare mutazioni con posizioni nuove e sicure delle stelle, si debbano considerare tutti di egual peso deducendone il medio, $\Delta \alpha=-0^{3} 56 \Delta \delta=-1.3$.

\title{
Karten zu 25 Veränderlichen in Aquila.
}

[Mit drei Tafeln].

Die hier abgedruckten 24 Karten stellen die Umgebung von 25 Variablen dar, welche von dem Unterzeichneten in A. N. 3925 und A. N. 3954 mitgeteilt worden sind. Sie sind durch das Mikroskop von den Platten nach dem Augenmaß abgezeichnet; und zwar vier von dem Unterzeichneten, alle ubrigen von Frau G. Wolf.

Auf den Kärtchen I bis 23 ist der Maßstab I' $=3.0 \mathrm{~mm}$; auf dem Kärtchen 24 dagegen mußte $I^{\prime}=2.2 \mathrm{~mm}$ genommen werden. Die letztere Abweichung war notwendig, weil sonst die 2 wei Variabeln $n$ und $o_{1}$ nicht mit einem Anschlußstern auf dieselbe Karte zu bringen gewesen wären. Dieses Kärt. chen verdient übrigens besonderes Interesse, weil es das nordwestliche Ende der stidlichsten Sternleere der *Tripelhöhle * darstellt. Beim Zeichnen der Kärtchen sind von uns die schwächsten Sterne nicht mitgenommen worden, weil es sonst zu mühsam oder wohl ganz unmöglich gewesen wäre, die in dieser Himmelsgegend sehr dicht stehenden Sterne alle in dem engen Kreis der Kärtchen unterzubringen. Trotzdem enthalten einige Kärtchen schon mehr als 200 Sterne in dem kleinen Gesichtsfeld.

Jedes Kärtchen trägt außer dem Variablen mindestens einen Bonner-Durchmusterungs-Stern, der zur Aufsuchung dienlich sein soll. Im folgenden seien die Bezeichnungen der Variablen und der Anschlußsterne zusammengestellt.

$$
\begin{aligned}
& \text { Karte var. Aquilae } \quad \alpha \text { I900.0 } \delta \text { I900.0 } \\
& \text { I } g=64 \cdot 1903 \\
& 2 \quad m_{1}=65.1903 \\
& 19^{\mathrm{h}} 27^{\mathrm{m}} 4^{\mathrm{s}} \cdot 78+10^{\circ} 18^{\prime} 39^{\prime \prime} \mathrm{I} \\
& \text { Schwankung } \\
& 1901-1903 \\
& 193025.8 \mathrm{I}+7214.3 \\
& \text { I I.5 - } 15 \\
& \text { I } 4-12.5 \\
& \text { I9 } 33 \text { II.20 +12 } 3345.5 \\
& <14-13 \\
& \text { I } \left.3-<14.5^{1}\right) \\
& \text { I } 4.5-x x^{1} \text { ) } \\
& 193420.15+\text { Ir } 435.0 \\
& 13-10.5 \\
& \text { I9 } 3556.75+94157.2 \\
& 14-I 1.5 \\
& \text { 19 } 3621.56+7 \text { II } 2.8 \\
& \text { I } 2.0-\quad 14.5 \\
& \text { I I }-<\text { I } 5 \\
& 194021.55+81211.7 \\
& <I_{5}-\mathrm{I} 3.5 \\
& * \mathrm{I}=\mathrm{BD},+10^{\circ} 3938 \\
& * \mathrm{I}=\mathrm{BD} .+6.4202 \\
& * 2=\mathrm{BD} .+6.4206 \\
& * \mathrm{I}=\mathrm{BD} .+12.3990 \\
& * \mathrm{I}=\mathrm{BD} .+\mathrm{I} 1.393^{\circ} \\
& * \mathrm{I}=\mathrm{BD} .+\mathrm{Ir} .393 \mathrm{I} \\
& * \mathrm{I}=\mathrm{BD} .+9.4202 \\
& * 2=\mathrm{BD} .+9.4204 \\
& * \mathrm{I}=\mathrm{BD} .+7.4182 \\
& * \mathrm{I}=\mathrm{BD} .+13.4122 \\
& * \mathrm{I}=\mathrm{BD} .+8.4204 \\
& * \mathrm{I}=\mathrm{BD} .+10.4043^{2} \text { ) }
\end{aligned}
$$

1) Bei Stern $d$ und $d_{1}$ sind die Schätzungen in A. N. 3925 verwechselt worden.

2) $\gamma$ Aquilae. 


\begin{tabular}{|c|c|c|c|}
\hline Karte & & var. & Aquilae \\
\hline I I & $f_{1}$ & $=$ & $73 \cdot 1903$ \\
\hline I 2 & $k_{1}$ & $=$ & $74 \cdot 1903$ \\
\hline I3 & $m$ & $=$ & $75 \quad 3903$ \\
\hline 14 & $g_{1}$ & $=$ & 76.1903 \\
\hline I 5 & $e$ & $=$ & $77 \cdot 1903$ \\
\hline I 6 & $c$ & $=$ & 78.1903 \\
\hline 17 & $a_{1}$ & $=$ & $79.19 \circ 3$ \\
\hline I 8 & $b$ & $=$ & 80.1903 \\
\hline I 9 & $i$ & $=$ & 81.1903 \\
\hline 20 & $f$ & $=$ & $82 \cdot 1903$ \\
\hline 21 & $h$ & $=$ & $83 \cdot 1903$ \\
\hline 22 & $i_{1}$ & $=$ & $84 \cdot 1903$ \\
\hline 23 & $l$ & $=$ & 85.1903 \\
\hline 24 & $n$ & $=1$ & I I I . I 904 \\
\hline${ }^{\star}$ & $o_{1}$ & $=$ & 112.1904 \\
\hline
\end{tabular}

\begin{tabular}{|c|c|c|c|c|c|}
\hline \multirow{2}{*}{\multicolumn{3}{|c|}{$\begin{array}{c}\alpha 1900.0 \\
I 9^{h} 4 I^{m} 56.00\end{array}$}} & \multicolumn{3}{|c|}{81900.0} \\
\hline & & & $+10^{\circ}$ & $13^{\prime}$ & 6". I \\
\hline I 9 & 42 & $22.6 \mathrm{I}$ & +7 & 23 & 3.6 \\
\hline I9 & 42 & 30.19 & +12 & I 4 & 19.5 \\
\hline 19 & 42 & $49 \cdot 3^{8}$ & +9 & $4 I$ & $5^{6.6}$ \\
\hline I9 & 43 & 39.78 & $+I I$ & 16 & 33.0 \\
\hline I9 & 44 & 34.78 & +12 & 7 & $7 \cdot 4$ \\
\hline 19 & 46 & $0.2 \mathrm{I}$ & +12 & 33 & 57.9 \\
\hline 19 & 46 & I 5.30 & +12 & $5^{8}$ & 0.0 \\
\hline 19 & 48 & $42.3^{8}$ & +9 & 6 & $37 \cdot 3$ \\
\hline 19 & 48 & $59 \cdot 37$ & +10 & 44 & 5.1 \\
\hline r 9 & 49 & 6.26 & +9 & 24 & 0.8 \\
\hline I9 & 49 & 24.82 & +7 & 21 & I. 4 \\
\hline r9 & 49 & 32.68 & +7 & 44 & 39.8 \\
\hline 19 & 33 & 40.66 & +10 & 22 & $2, I$ \\
\hline 19 & 34 & 12.46 & +10 & I 6 & $35 \cdot 7$ \\
\hline
\end{tabular}

\begin{tabular}{|c|c|c|}
\hline \multicolumn{3}{|c|}{$\begin{array}{l}\text { Schwankung } \\
\text { I901 - I903 }\end{array}$} \\
\hline$<I_{5}$ & - & I 4 \\
\hline I 3.5 & - & I I. 5 \\
\hline I 1.5 & $-<$ & I 5 \\
\hline$<\mathrm{I}_{5}$ & - & I 2.0 \\
\hline I 0.0 & - & 12.0 \\
\hline 11.0 & - & 13.0 \\
\hline 14 & - & 11.5 \\
\hline I I & $-<$ & 14 \\
\hline I 2 & - & 14 \\
\hline 12 & - & 13 \\
\hline I I & - & I 3 \\
\hline I 2.5 & - & II .5 \\
\hline 13 & $-<$ & \\
\hline I 3 & $-<$ & \\
\hline 12.7 & - & I I. 2 \\
\hline
\end{tabular}

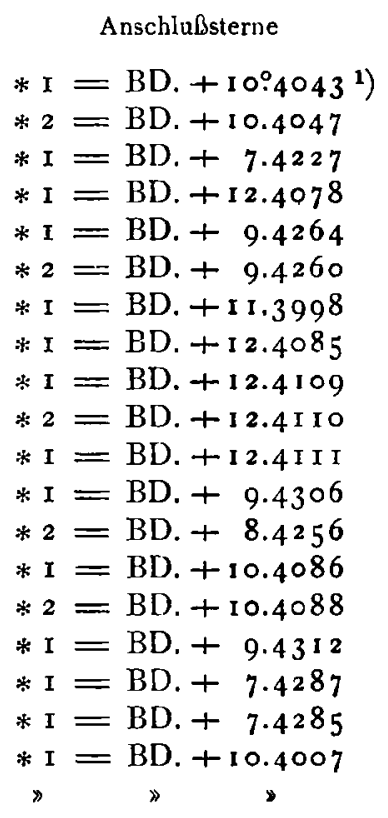

Max Wolf.

Gr. Astrophys. Observatorium Königstuhl-Heidelberg, I 904 Juni I.

\section{Beobachtungen des Sterns Gro. 1830.}

Wie voriges Jahr (Astr. Nachr. Nr. 3904) wurde der Stern auch heuer beobachtet und zwar:

\begin{tabular}{|c|c|c|c|c|c|c|c|c|}
\hline & & $\alpha 1904.0$ & E 1904.0 & & & & $\alpha I 904.0$ & 81904.0 \\
\hline $9 \circ 3$ & Dez. 2 & ${ }_{11}{ }^{\mathrm{h}} 47^{\mathrm{m}} 27^{\mathrm{s}} .08$ & $+38^{\circ} 24^{\prime} 26^{\prime \prime} 9$ & 1904 & April & 22 & $11^{\mathrm{h}} 47^{\mathrm{m}_{2} 7^{\mathrm{s}} \cdot 05}$ & $+38^{\circ} 24^{\prime} 27^{\prime \prime} \cdot 5$ \\
\hline 90. & April & 26.84 & 25.4 & 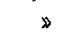 & $»$ & 23 & 27.07 & 26.5 \\
\hline » & $\gg \quad I$ & 26.94 & 26.1 & $»$ & 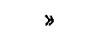 & 25 & 26.93 & 27.3 \\
\hline
\end{tabular}

Beobachter: Korvetten-Kapitän Koß.

Positionen der Berl. Jahrb.-Sterne mit den Auwersschen Verbesserungen; bei der Reduktion auf I904.0 wurde die Eigenbewegung aus Newcombs Catalogue of Fundamental Stars angewendet.

Pola, I904 April 30.

Der Vorstand: Koß, Korv.Kapt.

Ephemeride des Planeten (511) [1903 LU].

Die nachfolgende Ephemeride ist für $12^{\text {h }}$ M. Z. Berlin aus den Elementen im B. J. 1906 berechnet.

\begin{tabular}{|c|c|c|c|c|c|}
\hline \multicolumn{2}{|c|}{1904} & $\alpha$ app. & $\delta$ app. & \multirow{2}{*}{$\frac{\log r}{0.5488}$} & \multirow{2}{*}{$\begin{array}{c}\log A \\
0.4083\end{array}$} \\
\hline Juli & IO & $20^{\mathrm{h}} 39^{\mathrm{m}} 42^{\mathrm{s}}$ & $-23^{\circ} 211^{\prime}$ & & \\
\hline & 12 & 3820 & 2333.6 & & \\
\hline & $\mathrm{I}_{4}$ & 3656 & 2346.0 & 0.5482 & 0.4048 \\
\hline & 16 & $35 \quad 28$ & $23 \quad 5^{8.4}$ & & \\
\hline & I 8 & 3358 & $24 \quad 10.7$ & 0.5475 & 0.4020 \\
\hline & 20 & $32 \quad 27$ & 2423.0 & & \\
\hline & 22 & $30 \quad 54$ & 2435.1 & 0.5469 & 0.4000 \\
\hline & 24 & $29 \quad 19$ & 2447.1 & & \\
\hline 8 & 26 & 2744 & 2459.0 & 0.5462 & 0.3989 \\
\hline$\gamma$ & 28 & 268 & $25 \quad 10.7$ & & \\
\hline & 30 & 202432 & $-25 \quad 22.2$ & 0.5455 & $0.39^{85}$ \\
\hline
\end{tabular}

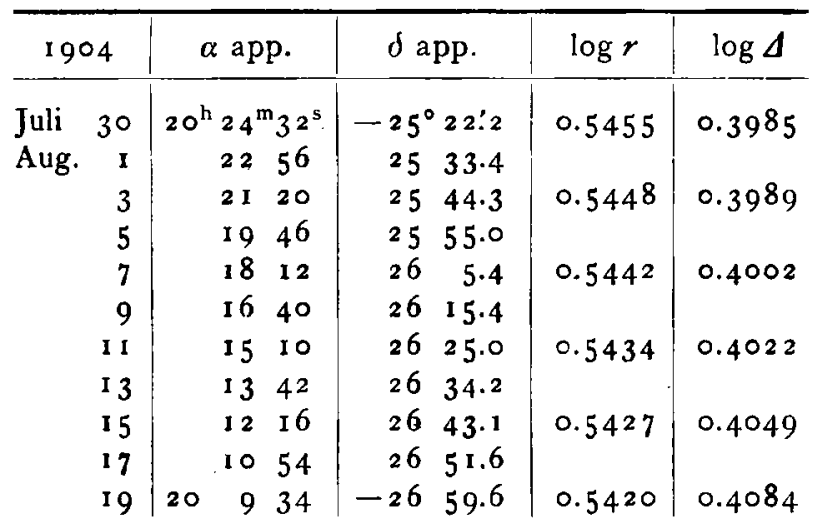

Gr. Io.I. Variation RA. $\pm \mathrm{I}^{\mathrm{m}}$ Dekl. $\pm 0: 7$. Präz. bis $1855.0-2^{\mathrm{m}} 55^{\mathrm{s}}-9: 8$.

Berlin-Halensee, I904 Juni.

A. Wegener. 